Pacific Journal of Mathematics

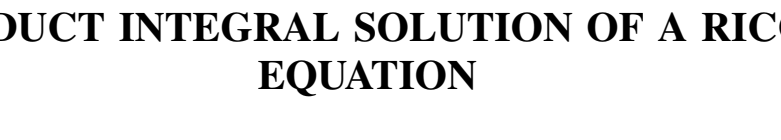




\title{
A PRODUCT INTEGRAL SOLUTION OF A RICCATI EQUATION
}

\author{
Burrell W. Helton
}

\section{In Memory of Professor H. S. Wall}

Product integrals are used to show that, if $d w, G, H$ and $K$ are functions from number pairs to a normed complete ring $N$ which are integrable and have bounded variation on $[a, b]$ and $v^{-1}$ exists and is bounded on $[a, b]$, then the integral equation

$$
\beta(x)=w(x)+(L R L R) \int_{a}^{x}(\beta H+G \beta+\beta K \beta)
$$

has a solution $\beta(x)=v^{-1}(x) u(x)$ on $[a, b]$, where $u$ and $v$ are defined by the matrix equation

$$
[u(x), v(x)]=[w(a), 1]_{a} \prod^{x}\left(I+\left[\begin{array}{ll}
H & -K \\
d w & -G
\end{array}\right]\right)
$$

The above results are used to show that if $p, q, h$ and $r$ are quasicontinuous functions from the numbers to $N$ such that $h$ is left continuous and has bounded variation and $p, q$ and $h$ commute, then the solution on $[a, b]$ of the differential-type equation $f^{* *}+f^{*} p+f q=r$ is

$$
f(x)=f(a){ }_{a} \prod^{x}(1-\beta d h)+(R) \int_{a}^{x} d z_{t} \prod^{x}(1-\beta d h),
$$

where $f(x)-f(a)=(L) \int_{a}^{x} f^{*} d h, \beta$ is the solution of

$$
\beta(x)=(L) \int_{a}^{x} q d h+(L L) \int_{a}^{x} \beta(-p d h)+(L R) \int_{a}^{x} \beta d h \beta,
$$

and $z$ is defined in terms of $p, q, r, h$ and $\beta$.

1. Introduction. Adam [1] introduced the concept of continuous continued fractions and showed that the solution of $y^{\prime}=$ $g^{\prime} y^{2}-f^{\prime}$ could be given as a continuous continued fraction, provided $f^{\prime}$ and $g^{\prime}$ are continuous and positive. Wall [11] [12] showed that, if $F_{11}, F_{12}, F_{21}$ and $F_{22}$ are continuous functions of bounded variation from the real numbers to the complex numbers and $|b-a|$ is sufficiently 
small, then the solution of

$$
w(x)=z+\int_{b}^{x} w^{2} d F_{21}+\int_{b}^{x} w d\left(F_{22}-F_{11}\right)-\int_{b}^{x} d F_{12}
$$

is $w(x)=\left[M_{11}(x, b) z+M_{12}(x, b)\right]\left[M_{21}(x, b) z+M_{22}(x, b)\right]^{-1}$, where $F=$ $\left[\begin{array}{ll}F_{11} & F_{12} \\ F_{21} & F_{22}\end{array}\right]$ and $\left[\begin{array}{ll}M_{11} & M_{12} \\ M_{21} & M_{22}\end{array}\right]$ is the function such that $M(x, y)=$ $1+\int_{x}^{y} M(x, s) d F(s)$. MacNerney, using the Stieltjes integral in [7] and the subdivision-refinement-type mean integral in [8], extended Wall's results to some types of quasicontinuous linear transformations and showed that the solution of Equation (1) can also be expressed as a continuous continued fraction [8, Theorem 5.3]. In this paper the product integral theory developed by MacNerney [8] [9] and the author [3] is used to find and express (in \$3) the solution of

$$
\beta(x)=w(x)+(L R L R) \int_{a}^{x}(\beta H+G \beta+\beta K \beta)
$$

and to find and express (in $\S 4$ ) the solution of

$$
f^{* *}+f^{*} p+f q=r
$$

where $w, p, q, r, G, H, K$ are quasicontinuous functions from numbers or pairs of numbers to a normed complete ring $N$.

2. Definitions and notations. The symbol $R$ denotes the set of real numbers and $N$ is a ring which has an identity element 1 and a norm $|\cdot|$ with respect to which $N$ is complete and $|\mathbf{1}|=1$ (henceforth, the symbol 1 will be used for this identity element). Functions from $R$ to $N$ and from $R \times R$ to $N$ will be represented by lower case letters and upper case letters, respectively. All sum and product integrals are subdivision-refinement-type limits. If $G$ is a function from $R \times R$ to $N$, the product integral of $G$ exists on $[a, b]$ iff there exists $A \in N$ such that if $\epsilon$ is a positive number then there is a subdivision $D$ of $[a, b]$ such that if $\left\{x_{i}\right\}_{0}^{n}$ is a refinement of $D$ then $\left|A-G_{1} G_{2} \cdots G_{n}\right|<\epsilon$, where $G_{i}=G\left(x_{i-1}, x_{1}\right)$ for $i=1,2, \cdots, n$. The symbol ${ }_{a} \Pi^{b} G$ will be used to represent the limit $A$. A similar definition holds for the sum integral. Upper case letters preceding an integral symbol show how the integrand is to be evaluated: i.e., $(L R L R) \int_{a}^{b}(f H+G f+f K f)=$ $\int_{a}^{b} M$, where for $x<y$ 


$$
M(x, y)=f(x) H(x, y)+G(x, y) f(y)+f(x) G(x, y) f(y) .
$$

Also, $G \in O A^{0}$ on $[a, b]$ iff $\int_{a}^{b} G$ exists and $\int_{a}^{b}\left|G-\int G\right|=0 ; G \in O M^{0}$ on $[a, b]$ iff ${ }_{x} \Pi^{y}(1+G)$ exists for $a \leqq x \leqq y \leqq b$ and $\int_{a}^{b}|(1+G)-\Pi(1+G)|=0 ; G \in O B^{0}$ on $[a, b]$ iff there is a number $M$ and a subdivision $D$ of $[a, b]$ such that, if $\left\{x_{i}\right\}_{0}^{n}$ is a refinement of $D$, then $\sum_{1}^{n}\left|G\left(x_{i-1}, x_{i}\right)\right| \leqq M$; the function $v^{-1}$ exists on $[a, b]$ means $v(x) v(x)^{-1}=$ $v(x)^{-1} v(x)=1$ for $x \in[a, b]$. The function $G^{-1}$ exists on $[a, b]$ means there is a subdivision $\left\{x_{i}\right\}_{0}^{n}$ of $[a, b]$ such that if $0<i \leqq n$ and $x_{i-1} \leqq x<$ $y \leqq x_{i}$, then $G(x, y)^{-1} G(x, y)=G(x, y) G(x, y)^{-1}=1$. If $\left\{x_{i}\right\}_{0}^{n}$ is a subdivision, the symbols $f_{i-1}, f_{i}$, and $G_{i}$ will be used as shorthand notations for $f\left(x_{i-1}\right), f\left(x_{i}\right)$ and $G\left(x_{i-1}, x_{i}\right)$, respectively. For additional details pertaining to these definitions, see [3], [4], and [9]. The main results of this paper will be designated as theorems; the supporting theorems will be labeled as lemmas.

3. A Riccati integral equation. In this section we derive a solution for the integral equation

$$
f(x)=w(x)+(L R L R) \int_{a}^{x}(f H+G f+f K f) .
$$

Since the $O A^{0}$ property plays an important role in this paper, please note that the function $G \in O A^{0}$ if at least one of the following conditions is satisfied:

(1) there is a function $g$ such that

$$
G(x, y)=g(y)-g(x)
$$

(2) if $G(x, y)=f(x) H(x, y)$, where $f$ is quasicontinuous and $H \in$ $O A^{0}$ and $O B^{0},[4$, Theorem 2];

(3) if $G$ is an integrable function from number pairs to a real Hilbert space which is finite dimensional, [2, Theorem 2].

Also note that, if $H, K, W, G$ are functions from $R \times R$ to $N$ which belong to $O A^{\circ}$ and $O B^{\circ}$, then $\left[\begin{array}{ll}H & K \\ W & G\end{array}\right]$ represents a matrix $Q$ such that $Q \in O A^{0}$ and $O B^{0}$ and, by Lemma 3.1, $Q \in O M^{0}$.

LEMMA 3.1. If $G$ is a function from $R \times R$ to a normed complete ring and $G \in O B^{0}$, then the following statements are equivalent:

(1) $G \in O A^{0}$ on $[a, b]$ and

(2) $G \in O M^{0}$ on $[a, b]$. 
This is Theorem 3.4 of [3].

THEOREM 3.2. Given. (1) $[a, b]$ is a number interval. (2) $w$ is $a$ function from $R$ to $N$ and $H, G$ and $K$ are functions from $R \times R$ to $N$ such that each of $d w, H, G$ and $K$ belongs to $O A^{0}$ and $O B^{0}$.

(3) $u$ and $v$ are functions from $R$ to $N$ such that if $x \in[a, b]$ then $u(x)$ and $v(x)$ are defined by the matrix equation

$$
[u(x), v(x)]=[w(a), 1]_{a} \Pi^{x}\left(I+\left[\begin{array}{ll}
H & -K \\
d w & -G
\end{array}\right]\right)
$$

and $v^{-1}$ exists and is bounded.

(4) $f$ is a bounded function from $R$ to $N, f(a)=w(a)$ and $f(x)=$ $v(x)^{-1} u(x)$ for $x \in[a, b]$.

Conclusion. If $x \in[a, b]$, then

$$
f(x)=w(x)+(L R L R) \int_{a}^{x}(f H+G f+f K f) .
$$

Furthermore, if $w$ is a constant function, then

$$
\begin{gathered}
f(x)=\left[\prod_{a}^{x}(1-G)-w(a)(L R) \int_{a}^{x} \prod^{t}(1+H) K_{t} \Pi^{x}(1-G)\right]^{-1} \\
{\left[w(a){ }_{a} \prod^{x}(1+H)\right] .}
\end{gathered}
$$

Proof. Let $Q$ be the function such that $Q=\left[\begin{array}{ll}1+H & -K \\ d w & 1-G\end{array}\right]$; then $Q-I \in O A^{0}$ and $O B^{0}$ and, by Lemma 3.1, $Q-1 \in O M^{0}$. Suppose $x \in(a, b]$ and $\left\{x_{i}\right\}_{0}^{n}$ is a subdivision of $[a, x]$. If $0<i \leqq n$, then there exist $a_{i}$ and $b_{i} \in N$ such that

$$
\begin{aligned}
& {\left[v\left(x_{i}\right) f\left(x_{i}\right), v\left(x_{i}\right)\right]=\left[u\left(x_{i}\right), v\left(x_{i}\right)\right]} \\
& \quad=[w(a), 1]_{a} \prod^{x_{i-1}} Q_{x_{i-1}} \prod^{x_{i}} Q \\
& \quad=\left[u\left(x_{i-1}\right), v\left(x_{i-1}\right)\right]_{x_{i-1}} \prod^{x_{i}}\left[\begin{array}{lr}
1+H & -K \\
d w & 1-G
\end{array}\right] \\
& =\left[u_{i-1}, v_{i-1}\right]\left[\begin{array}{cr}
1+H_{i} & -K_{i} \\
\Delta w_{i} & 1-G_{i}
\end{array}\right]+\left[a_{i}, b_{i}\right] \\
& =v_{i-1}\left[f_{i-1}, 1\right]\left[\begin{array}{cr}
1+H_{i} & -K_{i} \\
\Delta w_{i} & 1-G_{i}
\end{array}\right]+\left[a_{i}, b_{i}\right]
\end{aligned}
$$




$$
=v_{\imath-1}\left[f_{i-1}\left(1+H_{i}\right)+\Delta w_{i},-f_{i-1} K_{t}+(1-G)\right]+\left[a_{i}, b_{i}\right] .
$$

Therefore,

$$
\left(v^{-1}{ }_{1-1} v_{1}\right) f_{i}=f_{i-1}\left(1+H_{i}\right)+\Delta w_{1}+v^{-1}{ }_{1-1} a_{i}
$$

and

$$
v^{-1}{ }_{1-1} v_{1}=-f_{i-1} K_{i}+1-G_{i}+v^{-1}{ }_{i-1} b_{i}
$$

hence,

$$
\left(-f_{i-1} K_{i}+1-G_{i}+v^{-1}{ }_{i-1} b_{i}\right) f_{i}=f_{i-1}\left(1+H_{i}\right)+\Delta w_{i}+v^{-1}{ }_{i-1} a_{i}
$$

and

$$
f_{i}-f_{t-1}=\Delta w_{i}+f_{t-1} H_{t}+G_{i} f_{i}+f_{i-1} K_{l} f_{i}-v^{-1}{ }_{i-1} b_{l} f_{i}+v^{-1}{ }_{i-1} a_{i} .
$$

Since $f, u, v$ and $v^{-1}$ are bounded and since $\sum_{1}^{n}\left(\left|a_{i}\right|+\left|b_{i}\right|\right)$ can be made arbitrarily small with an appropriate choice of a subdivision (since $\left.Q \in O M^{0}\right)$, then the following integral exists and

$$
f(x)-f(a)=w(x)-f(a)+(L R L R) \int_{a}^{x}(f H+G f+f K f) .
$$

Since

$$
\prod_{1}^{n}\left[\begin{array}{ll}
p_{\imath} & q_{1} \\
0 & r_{\imath}
\end{array}\right]=\left[\begin{array}{ll}
p & q \\
0 & r
\end{array}\right]
$$

where $p=\prod_{1}^{n} p_{i}, q=\sum_{j=1}^{n}\left(\prod_{i=1}^{j-1} p_{i}\right) q_{j}\left(\prod_{i=j+1}{ }^{n} r_{i}\right)$ and $r=\prod_{i=1}{ }^{n} r_{t}$, and since all the following integrals and product integrals exist, then

$$
[w(a), 1] \text { a } \prod^{\prime}\left[\begin{array}{lr}
1+H & -K \\
0 & 1-G
\end{array} \mid=[w(a), 1]\left[\begin{array}{cc}
A & B \\
0 & D
\end{array}\right],\right.
$$

where $\quad A={ }_{a} \Pi^{\prime}(1+H), \quad B=(L R) \int_{a}^{x}\left[{ }_{a} \Pi^{t}(+H)\right](1-K)\left[\Pi_{t} \Pi^{x}(1-G)\right]$ and $D={ }_{a} \Pi^{\prime}(1-G)$; hence, if $w$ is a constant function, then

$$
f(x)=[w(a) B+D]^{-1}[w(a) A] .
$$

THEOREM 3.3. Given. (1) $[a, b]$ is a number interval;

(2) $w$ is a function from $R$ to $N$ and $H, G$ and $K$ are functions from 
$R \times R$ to $N$ such that each of $d w, H, G$ and $K$ belongs to $O A^{0}$ and $O B^{0}$;

(3) $u$ and $v$ are functions from $R$ to $N$ such that, if $x \in[a, b]$, then $u(x)$ and $v(x)$ are defined by the matrix equation

$$
[u(x), v(x)]=[w(a), 1]_{a} \prod^{x}\left(I+\left[\begin{array}{ll}
H & -K \\
d w & -G
\end{array}\right]\right)
$$

and $v(x)^{-1}$ exists;

(4) $f$ is a bounded function from $R$ to $N, f(a)=w(a)$, $\left(1-G_{i}-f_{i-1} K_{t}\right)^{-1}$ exists and

$$
f(x)=w(x)+(L R L R) \int_{a}^{x}(f H+G f+f K f)
$$

for $x \in[a, b]$.

Conclusion. If $x \in[a, b]$, then $f(x)=v(x)^{-1} u(x)$.

Proof. Suppose $x \in[a, b]$ and $\left\{x_{i}\right\}_{0}^{n}$ is a subdivision of $[a, b]$. If $0<i \leqq n$, then there exists $\epsilon_{i} \in N$ such that

$$
\begin{aligned}
f\left(x_{i}\right) & =w\left(x_{i}\right)+(L R L R) \int_{a}^{x_{i}}(f H+G f+f K f) \\
& =\Delta w_{i}+f_{i-1}+f_{i-1} H_{i}+G_{i} f_{i}+f_{i-1} K_{i} f_{i}+\epsilon_{i}
\end{aligned}
$$

and $f_{i}=b_{i}^{-1} a_{i}$, where $b_{i}=1-G_{i}-f_{i-1} K_{i}$ and $a_{i}=$ $f_{i-1}\left(1+H_{i}\right)+\left(\Delta w_{i}+\epsilon_{i}\right)$. For $i=1,2,3, \cdots, n$, let $R_{i}$ be the $2 \times 2$ matrix $R_{\imath}=\left[\begin{array}{cc}1+H_{i} & -K_{i} \\ \Delta w_{i}+\epsilon_{i} & 1-G_{i}\end{array}\right]$; let $a_{0}=w(a)$ and $b_{0}=1$; then $\left\{a_{i}\right\}_{0}^{n}$ and $\left\{b_{i}\right\}_{0}^{n}$ are elements of $N$ such that, if $0<i \leqq n$, then $f_{i}=b_{i}^{-1} a_{i}$ and

$$
\left[a_{i}, b_{i}\right]=\left[f_{i-1}, 1\right] R_{i}=\left[b_{i-1}^{-1} a_{i-1}, 1\right] R_{i}=b_{i-1}^{-1}\left[a_{i-1}, b_{i-1}\right] R_{i}
$$

Therefore

$$
\left[a_{n}, b_{n}\right]=\left(\prod_{i=n}^{1} b_{i-1}^{-1}\right)\left[f_{0}, 1\right] \prod_{i=1}^{n} R_{i}
$$

and

$$
\left(\prod_{i=1}^{n} b_{i-1}\right) b_{n}\left[f_{n}, 1\right]=\prod_{i=1}^{n} b_{i-1}\left[a_{n}, b_{n}\right]=\left[f_{0}, 1\right] \prod_{i=1}^{n} R_{i}
$$


Let $Q$ be the function from $R \times R$ to the set of $2 \times 2$ matrices such that $Q=\left[\begin{array}{cc}1+H & -K \\ d w & 1-G\end{array}\right]$. Since $f$ is quasicontinuous and since each of $d w, H, G$ and $K$ belong to $O A^{0}$ and $O B^{0}$, then $Q-I$ and $-G-f K \in$ $O A^{0}$ and $O B^{0}$ and it follows from Lemma 3.1 that $Q-I$ and $-G-f K$ belong to $O M^{0}$, the corresponding product integrals exist, $\int_{a}^{b}|Q-\Pi Q|=$ 0 and $\int_{a}^{b}|(1-G-f K)-\Pi(1-G-f K)|=0$. For each subdivision $\left\{x_{i}\right\}_{0}^{n}$ of [ $a, x]$, there exist elements $d_{1}, d_{2}$, and $d_{3}$ such that Equation (1) can be rewritten

$$
\left\{(L){ }_{a} \Pi^{x}(1-G-f K)+d_{1}\right\}\left[f_{n}, 1\right]=\left[f_{0}, 1\right]\left(\prod_{a}^{x} Q+d_{2}+d_{3}\right),
$$

where $1-G_{i}-f_{i-1} K_{i}$ is playing the role of $b_{i}$ and

$$
\begin{aligned}
& d_{1}=\prod_{i=1}^{n}\left(1-G_{i}-f_{i-1} K_{i}\right)-(L){ }_{a} \prod^{\lambda}(1-G-f K), \\
& d_{2}=\prod_{i=1}^{n} Q_{i}-{ }_{a} \prod^{\lambda} Q
\end{aligned}
$$

and

$$
d_{3}=\prod_{i=1}^{n} R_{i}-\prod_{i=1}^{n} Q_{i}=\sum_{i=1}^{n}\left(\prod_{j=1}^{i-1} Q_{j}\right)\left(R_{i}-Q_{i}\right) \prod_{j=i+1}^{n} R_{j}
$$

Since $R_{i}-Q_{i}=\left[\begin{array}{ll}0 & 0 \\ \epsilon_{i} & 0\end{array}\right]$, it follows from the $O M^{0}$ and $O A^{0}$ properties that each of $\left|d_{i}\right|,\left|d_{2}\right|$ and $\left|d_{3}\right|$ can be made arbitrarily small; hence $(L){ }_{a} \Pi^{x}(1-G-f K)[f(x), 1]=\left[f_{0}, 1\right]_{a} \Pi^{x} Q=[u(x), v(x)]$. It follows from the meaning of equality for matrices that $(L){ }_{a} \Pi^{x}(1-G-$ $f K)=v(x), v(x) f(x)=u(x)$ and $f(x)=v(x)^{-1} u(x)$.

LemmA 3.4. If $G \in O B^{0}$ on $[a, b]$ and $\epsilon>0$, then there is a number $p \in(a, b]$ such that, if $\left\{x_{i}\right\}_{0}^{n}$ is a subdivision of $[a, p]$, then $\sum_{2}^{n}\left|G_{i}\right|<\epsilon$.

Theorem 3.5. Given. $H, W, K$ and $G$ are functions from $R \times R$ to $N$ such that each of $H, W, K$ and $G$ belongs to $O A^{0}$ and $O B^{0}$ on $[a, b]$ and $u$ and $v$ are functions from $R$ to $N$ and are defined by the matrix equation

$$
[u(x), v(x)]=[u(a), v(a)]_{a} \prod^{\lambda}\left(I+\left\lceil\begin{array}{cc}
H & W \\
K & G
\end{array}\right\rceil\right)
$$


for $x \in[a, b]$. Conclusion. (1) If $p \in(a, b]$ and $0<k<1$ and $|v(a)-1|+\sum_{1}^{n}\left|u_{i-1} W_{i}+v_{i-1} G_{i}\right|<k$ for each subdivision $\left\{x_{i}\right\}_{0}^{n}$ of $[a, p]$, then $v^{-1}$ exist: and is bounded on $[a, p]$. (2) If $|v(a)-1|+$ $\left|u(a) W\left(a, a^{+}\right)+v(a) G\left(a, a^{+}\right)\right|<1$, then there exists $p \in(a, b]$ such that $v^{-1}$ exists and is bounded on $[a, p]$.

Proof. Since $H, W, K$ and $G \in O A^{0}$ and $O B^{0}$ on $[a, b]$, then $\left[\begin{array}{ll}H & W \\ K & G\end{array}\right] \in O A^{0}$ and $O B^{0}$ on $[a, b]$ and, by Lemma 3.1, $\left[\begin{array}{ll}H & W \\ K & G\end{array}\right] \in$ $O M^{0}$ on $[a, b]$; also, $u$ and $v$ are quasicontinuous and bounded on $[a, b]$.

We now prove Conclusion 1. Let $x \in[a, p]$ and let $\left\{x_{i}\right\}_{1}^{n}$ be a subdivision of $[a, x]$. For $i=1,2, \cdots, n$, there exist $a_{i}$ and $b_{i} \in N$ such that

$$
\begin{aligned}
{\left[u\left(x_{i}\right), v\left(x_{i}\right)\right] } & =[u(a), v(a)]_{a} \prod^{x_{i}}\left(I+\left[\begin{array}{cc}
H & W \\
K & G
\end{array}\right]\right) \\
& =\left[u_{i-1}, v_{i-1}\right]_{x_{i}-1} \prod^{x_{i}}\left(I+\left[\begin{array}{cc}
H & W \\
K & G
\end{array}\right]\right) \\
& =\left[u_{i-1}, v_{i-1}\right]\left[\begin{array}{cc}
1+H_{i} & W_{i} \\
K_{i} & 1+G_{i}
\end{array}\right]+\left[a_{i}, b_{i}\right] \\
& =\left[u_{i-1}\left(1+H_{i}\right)+v_{i-1} K_{i}, u_{i-1} W_{i}+v_{i-1}+v_{i-1} G_{i}\right]+\left[a_{i}, b_{i}\right]
\end{aligned}
$$

and

$$
v_{i}-1=\left(v_{i-1}-1\right)+u_{i-1} W_{i}+v_{i-1} G_{i}+b_{i}
$$

hence, by iteration and the norm properties,

$$
\begin{gathered}
|v(x)-1|=\left|v_{n}-1\right| \leqq\left|v_{0}-1\right|+\sum_{1}^{n}\left|u_{i-1} W_{i}+v_{i-1} G_{i}\right|+\sum_{1}^{n}\left|b_{i}\right| \\
<k+\sum_{1}^{n}\left|b_{i}\right| .
\end{gathered}
$$

Let $r=(k+1) / 2$. Since $\left[\begin{array}{ll}H & W \\ K & G\end{array}\right] \in O M^{0}$ and $u$ and $v$ are bounded on $[a, b]$, then there is a subdivision $\left\{x_{i}\right\}_{0}^{n}$ of $[a, x]$ such that $\sum_{1}^{n}\left|b_{i}\right|<r-k$ and, hence, $|v(x)-1|<r<1$. Let $v$ denote $v(x)$; then $v=1+(v-1)$, $v^{-1}$ exists, and

$$
v^{-1}=1-(v-1)+(v-1)^{2}-(v-1)^{3}+\cdots
$$




$$
\left|v^{-1}\right| \leqq(1-|v-1|)^{-1} \leqq(1-r)^{-1}
$$

Therefore, $v^{-1}$ exists and is bounded by $[1-(k+1) / 2]^{-1}$ on $[a, p]$.

Since $u$ and $v$ are bounded and $G$ and $W \in O B^{0}$ on $[a, b]$, then there exist numbers $p$ and $k$ satisfying Conclusion 1 , provided $\mid v(a)-$ $1|+| u(a) W\left(a, a^{+}\right)+v(a) G\left(a, a^{+}\right) \mid<1$; hence, Conclusion 2 follows as a corollary to Conclusion 1.

LEMMA 3.6. If $G$ is a function from $R \times R$ to $N$ such that $G \in O A^{0}$ and $O B^{\circ}$, then $|G| \in O A^{0}$.

A proof for this lemma is given in [6].

LEMmA 3.7. If $G$ is a function from $R \times R$ to $N$, and $G \in O A^{0}$ and $O B^{0}$, then $\left|\int_{a}^{b} G\right| \leqq \int_{a}^{b}|G|$.

Outline of proof.

$$
\left|\int_{a}^{b} G\right| \leqq \sum_{1}^{n}\left|\int_{x_{t,}}^{x_{i}} G-G_{i}\right|+\sum_{i}^{n}\left|G_{i}\right| .
$$

Lemma 3.8. Given. $H$ and $G$ are functions from $R \times R$ to $R$ and $c$ is a number such that $H \geqq 0, G \geqq 0,1-G \geqq c>0$, and $H$ and $G \in O A^{0}$ and $O B^{0}$ on $[a, b] ; f$ is a bounded function from $R$ to $R$ and $k$ is $a$ number such that $f(x) \leqq k+(L R) \int_{a}^{x}(f H+f G)$ for $x \in[a, b]$.

Conclusion. If $x \in[a, b]$, then $f(x) \leqq k_{a} \Pi^{x}(1+H)(1-G)^{-1}$. This is Theorem 4 of [4].

LEMMA 3.9. If $G \in O A^{0}$ and $O B^{0}$ and $f$ is quasicontinuous on $[a, b]$, then $f G$ and $G f \in O A^{0}$ on $[a, b]$.

This is a special case of $[4$, Theorem 2].

THEOREM 3.10. Given. (1) $[a, b]$ is a number interval;

(2) $\quad w$ is a function from $R$ to $N$ and $H, G$ and $K$ are functions from $R \times R$ to $N$ such that each of $d w, H, G$ and $K$ belongs to $O A^{0}$ and $O B^{0}$ on $[a, b]$;

(3) $f$ and $g$ are bounded functions from $R$ to $N$ and $c$ is a number such that $1-|B| \geqq c>0$, where $B(x, y)=G(x, y)+g(x) K(x, y)$ and on $[a, b]$ each of $f$ and $g$ is a solution of the integral equation 


$$
f(x)=w(x)+(L R L R) \int_{a}^{x}(f H+G f+f K f) .
$$

Conclusion. If $x \in[a, b]$, then $f(x)=g(x)$.

Proof. Since $f$ and $g$ are bounded and since $d w, H, G$ and $K \in$ $O A^{0}$ and $O B^{0}$, then each of $f, g$ and $|f-g|$ is a quasicontinuous function. Let $A$ be the function $A(x, y)=H(x, y)+K(x, y) f(y)$ for $a \leqq x<y \leqq b$; then it follows from Lemmas 3.6 and 3.9 that $A, B,|A|$ and $|B| \in O A^{0}$ and $O B^{0}$ and that $(L R) \int_{a}^{b}[|f-g||A|+|B||f-g|]$ exists. If $x \in[a, b]$, then

$$
\begin{aligned}
& |f(x)-g(x)|=\left|(L R) \int_{a}^{x}[(f-g) A+B(f-g)]\right| \\
& \leqq 0+(L R) \int_{a}^{x}[|f-g||A|+|B||f-g|] \quad \text { (Lemma 3.7). }
\end{aligned}
$$

It follows from Lemma 3.8 that

$$
|f(x)-g(x)| \leqq 0 \cdot{ }_{a} \Pi^{x}(1+|A|)(1-|B|)^{-1}=0 .
$$

Therefore, if $x \in[a, b]$, then $f(x)=g(x)$.

The restrictions $1-|B| \geqq c>0$ and $\left(1-G_{i}-f_{i-1} K_{i}\right)^{-1}$ cannot be deleted from the hypothesis of Theorem 3.10 and Theorem 3.3, respectively. Consider the following example. Let $u, v$, and $g$ be functions from $R$ to $R$ such that $u(x)=0$ for $x \in[0,2], v(x)=g(x)=0$ for $x \in[0,1]$ and $v(x)=g(x)=1$ for $x \in(1,2]$. Each of $u$ and $v$ is a solution on [0,2] for the equation $f(x)=(R) \int_{0}^{x} f d g$. See [5] for solutions of equations in which the restriction $1-|B| \geqq c>0$ does not hold.

Theorems similar to Theorems 3.2, 3.3 and 3.10 can be proved for $f(x)=u(x) v(x)^{-1}$,

$$
f(x)=w(x)+(R L R L) \int_{a}^{x}(f G+H f+f K f),
$$

and

$$
\left[\begin{array}{l}
u(x) \\
v(x)
\end{array}\right]={ }_{a} \prod^{x} Q\left[\begin{array}{c}
w(a) \\
1
\end{array}\right]
$$

where $Q=\left[\begin{array}{cc}1+H & d w \\ -K & 1-G\end{array}\right]$ and

$$
{ }_{a} \prod^{x} Q=\lim Q\left(x_{n-1}, x_{n}\right) \cdots Q\left(x_{1}, x_{2}\right) Q\left(x_{0}, x_{1}\right) \text {. }
$$


We will now compare the Riccati equation for Riemann-Stieltjes integrals with the Riccati equation for the $(L R L R)$-integral. In this and the next paragraph, $G$ is continuous at $p$ means $G\left(p^{-}, p\right)=0=$ $G\left(p, p^{+}\right)$; also, the symbol $(R S) \int_{a}^{b} E(f)$ is used to denote a RiemannStieltjes-type integral: i.e., for each subdivision $\left\{x_{i}\right\}_{0}^{n}$ of $[a, b]$, the approximating sum has the form $\Sigma_{1}^{n} E\left[f\left(c_{i}\right)\right]$, where $x_{i-1} \leqq c_{i} \leqq x_{i}$ for $i=1,2, \cdots, n$. Suppose that $w, H, G$ and $K$ satisfy the hypothesis of Theorem 3.2. If $f$ is the solution of the Riccati equation

$$
f(x)=w(x)+(R S) \int_{a}^{x} f H+(R S) \int_{a}^{x} G f+(R S) \int_{a}^{x} f K f
$$

on $[a, b]$, then $f$ is the solution of

$$
f(x)=w(x)+(L R L R) \int_{a}^{x}(f H+G f+f K f)
$$

on $[a, b]$. If $f$ is a solution of

$$
f(x)=w(x)+(R S) \int_{a}^{x}(f H+G f+f K f)
$$

on $[a, b]$ and either $f$ is continuous on $[a, b]$ or each of $H, G$ and $K$ is continuous on $[a, b]$, then $f$ is the solution of Equation 1 on $[a, b]$. Equation 2 can have a solution $f$ on $[a, b]$ even though each of $f, w, H, G$ and $K$ has a discontinuity.

Example. Suppose that $N$ is a field, $a<p \leqq b$, and $g$ is a function of bounded variation which is continuous on $[a, p)$ and on $[p, b] ; f$ is the function such that

$$
f(x)=1+(L R L R) \int_{a}^{x}(f d g+d g f+f d g f)
$$

for $x \in[a, p)$ and

$$
f(x)=-2-f\left(p^{-}\right)+(L R L R) \int_{p}^{x}(f d g+d g f+f d g f)
$$

for $x \in[p, b]$; also,

$$
g(p)-g\left(p^{-}\right)=-2\left[1+f\left(p^{-}\right)\right] / f\left(p^{-}\right)\left[f\left(p^{-}\right)+2\right] .
$$


The function $f$ is the solution on $[a, b]$ of Equation (2) with $d g=H=$ $G=K$; however, $f$ is not the solution of Equation (1) unless $f\left(p^{-}\right)=$ - 1. Furthermore, if $g(p)$ is defined differently, then Equation (2) has no solution on $[a, p]$.

In order for the Riemann-Stieltjes equation to have a solution which is not a solution of the ( $L R L R)$-equation, there must be an interdependence between the functions $w, H, G$ and $K$. The following discussion illustrates this. Suppose that $N$ is a field and that $w, H, G$ and $K$ are functions that satisfy the hypothesis of Theorem 3.2 and that on $[a, b]$ the function $f$ is a solution of Equation (2) but is not a solution of Equation (1); then there is a number $p \in[a, b]$ such that $f$ is not continuous at $p$. For convenience suppose that $f\left(p^{-}\right) \neq f(p)$ and, in the following manipulations, let $f_{1}, f_{2}, \Delta w, H, G$ and $K$ denote $f\left(p^{-}\right), f(p)$, $w(p)-w\left(p^{-}\right), H\left(p^{-}, p\right), G\left(p^{-}, p\right)$ and $K\left(p^{-}, p\right)$, respectively. Then

$$
\begin{aligned}
f(p) & =f\left(p^{-}\right)+\Delta w+(R S) \int_{p^{-}}^{p}(f H+G f+f K f), \\
f_{2} & =f_{1}+\Delta w+f_{1} H+G f_{1}+f_{1} K f_{1}, \\
& =f_{1}+\Delta w+f_{2} H+G f_{2}+f_{2} K f_{2}, \\
& f_{2} H+G f_{2}+f_{2} K f_{2}=f_{1} H+G f_{1}+f_{1} K f_{1}
\end{aligned}
$$

and

$$
\left(f_{2}-f_{1}\right)\left(H+K f_{2}\right)+\left(G+f_{1} K\right)\left(f_{2}-f_{1}\right)=0 .
$$

Since $f_{2}-f_{1} \neq 0$ and $N$ is a field, then

$$
H+G+K f_{2}+f_{1} K=0 .
$$

Substituting for $f_{2}$ and simplifying, we obtain

$$
K^{2} f_{1}^{2}+(2+H+G) K f_{1}+(H+G+\Delta w K)=0 .
$$

Since $f_{1}=f\left(p^{-}\right)=w\left(p^{-}\right)+(R S) \int_{a}^{p^{-}}(f H+G f+f K f)$, then the value of $f\left(p^{-}\right)$depends only on the values of $w, H, G$ and $K$ on the half open interval $[a, p)$; however, Equation (3) depends on the values of $w, H, G$ and $K$ on the closed interval $[a, p]$. Hence, these functions cannot be defined independently. For example, if $K \neq 0$ and a different value is assigned to $w(p)$, then Equation (3) is no longer true and the RiemannStieltjes equation has no solution on $[a, p]$ unless compensating values are assigned to $H\left(p^{-}, p\right), G\left(p^{-}, p\right)$ and $K\left(p^{-}, p\right)$. However, the new $(L R L R)$-Riccati equation will have a solution on $[a, p]$. 
4. A differential-type equation. In this section we find the solution of $f^{* *}+f^{*} p+f q=r$, where $f^{*}$ and $f^{* *}$ are defined as follows. If $[a, b]$ is a number interval and $h$ is a left continuous function from $R$ to $N$ such that $d h \in O B^{0}$, then $D(h, a, b)$ denotes the set of ordered pairs of functions such that $(f, g) \in D(h, a, b)$ iff $g$ is a quasicontinuous function from $R$ to $N$ such that $f(x)-f(a)=$ (L) $\int_{a}^{x} g d h$ for $x \in[a, b]$. If $(f, g) \in D(h, a, b)$, then $g$ is denoted by $f^{*}$. Also,

$f^{* *}=\left(f^{*}\right)^{*}$ and $f \cong w$ iff $(L) \int_{a}^{x} f d h=(L) \int_{a}^{x} w d h$ for $x \in[a, b]$. In this section all integrals and product integrals are Cauchy-left-type integrals unless indicated otherwise.

Lemma 4.1. If $\left(f, f^{*}\right)$ and $\left(g, g^{*}\right) \in D(h, a, b)$, then $\left(f+g, f^{*}+g^{*}\right) \in D(h, a, b)$.

Lemma 4.2. If $\left(f, f^{*}\right)$ and $\left(g, g^{*}\right) \in D(h, a, b), g^{*}, h$ and $g$ commute and $z$ is the function such that $z(x)=g\left(x^{+}\right)-g(x)$ for $x \in[a, b]$, then $\left(f g, f^{*} g+f g^{*}+f^{*} z\right) \in D(h, a, b)$.

Indication of proof. Since $\left(g, g^{*}\right)$ and $\left(f, f^{*}\right) \in D(h, a, b)$, then $g$ is left continuous and $d f \in O B^{0}$; hence,

$$
\begin{aligned}
\int_{a}^{x} d f d g & =(L) \int_{a}^{x}(d f) z, \\
(L) \int_{a}^{x}(d f) g & =(R) \int_{a}^{x}[(d f) g-(d f)(d g)]
\end{aligned}
$$

and

$$
\text { (L) } \begin{aligned}
\int_{a}^{x}\left(f^{*} g+f g^{*}+f^{*} z\right) d h & =(L L L) \int_{a}^{x}[(d f) g+f d g+(d f) z] \\
& =(R L L) \int_{a}^{x}[(d f) g+f d g-(d f) d g+(d f) z] \\
& =(R L) \int_{a}^{x}[(d f) g+f d g] \\
& =f(x) g(x)-f(a) g(a)
\end{aligned}
$$

Lemma 4.3. Given. $[a, b]$ is a number interval; $f$ and $h$ are functions from $R$ to $N$ such that $f(a)=h(a)$ and $d h \in O B^{0} ; G$ is a function from $R \times R$ to $N$ such that $G \in O B^{0}$ and $O A^{0}$ 
Conclusion. The following statements are equivalent:

(1) if $x \in[a, b]$, then $f(x)=h(x)+(L) \int_{a}^{x} f G$; and

(2) if $x \in[a, b]$, then

$$
f(x)=f(a){ }_{a} \Pi^{x}(1+G)+(R) \int_{a}^{x} d h_{t} \Pi^{x}(1+G) .
$$

This lemma is a special case of Theorem 5.1 of [3].

THEOREM 4.4. Given. (1) $[a, b]$ is a number interval; (2) $h, p, q, u, v, \beta$ and $s$ are functions from $R$ to $N$ such that $h$ is left continuous, dh $\in O B^{\circ}, p$ and $q$ are quasicontinuous on $[a, b]$ and, if $x \in[a, b]$, then $u(x)$ and $v(x)$ are defined by the matrix equation

$$
[u(x), v(x)]=[0,1](\dot{L}){ }_{a} \prod^{x}\left(I+\left[\begin{array}{rr}
-p & -1 \\
q & 0
\end{array}\right] d h\right),
$$

$v(x)^{-1}$ exists, $\beta(x)=v(x)^{-1} u(x)$ and $s(x)=\beta\left(x^{+}\right)-\beta(x) ;$ also, $v^{-1}$ is bounded on $[a, b]$; (3) if $a \leqq x \leqq y \leqq b$, then $p(x), p(y), q(x), q(y)$, $h(x)$ and $h(y)$ commute; (4) $f$ and $r$ are functions from $R$ to $N$ and $r$ is quasicontinuous.

Conclusion. The following statements are equivalent.

(1) There exist functions $f^{*}$ and $f^{* *}$ such that $\left(f, f^{*}\right)$ and $\left(f^{*}, f^{* *}\right) \in D(h, a, b)$ and such that on $[a, b]$

$$
f^{* *}+f^{*} p+f q=r .
$$

(2) If $x \in[a, b]$, then

$$
f(x)=f(a)(L){ }_{a} \prod^{x}(1-\beta d h)+(R) \int_{a}^{x} d z(L){ }_{t} \prod^{x}(1-\beta d h),
$$

where $\quad \alpha=p-\beta-s, \quad z(x)=f(a)+(L) \int_{a}^{x} w d h, \quad g(x)=$ $f^{*}(a)+(L) \int_{a}^{x} r d h$ and

$$
w(x)=f^{*}(a)(L){ }_{a} \prod^{x}(1-\alpha d h)+(R) \int_{a}^{x} d g(L){ }_{t} \prod^{x}(1-\alpha d h) .
$$

Proof. Since $d h \in O B^{\circ}$ and $h$ is left continuous and since $p$ and $q$ are quasicontinuous, then $u$ and $v$ are left continuous and 
quasicontinuous. Since $v^{-1}$ is bounded and $\beta=v^{-1} u$, then $\beta$ is left continuous, quasicontinuous and commutes with $h$. If $x \in[a, b]$, it follows from Theorem 3.2 that

$$
\beta(x)=(L) \int_{a}^{x} q d h+(L L) \int_{a}^{x} \beta(-p d h)+(L R) \int_{a}^{x} \beta d h \beta .
$$

Let $\alpha, s$ and $k$ be the functions such that $s(t)=\beta\left(t^{+}\right)-\beta(t), \alpha=$ $p-\beta-s, k(a)=0$, and $k=q+\beta^{2}-\beta p+\beta s$; then, for $x \in[a, b]$,

$$
\begin{aligned}
(L) \int_{a}^{x} k d h= & (L) \int_{a}^{x}\left(q+\beta^{2}-\beta p+\beta s\right) d h \\
= & (L) \int_{a}^{x} q d h+\left[(L R) \int_{a}^{x} \beta d h \beta-(L) \int_{a}^{x} \beta d h d \beta\right] \\
& +(L L) \int_{a}^{x} \beta(-p d h)+(L L) \int_{a}^{x} \beta s d h .
\end{aligned}
$$

Since $\beta$ is left continuous, then

$$
\text { (L) } \int_{a}^{x} \beta d h d \beta=(L L) \int_{a}^{x} \beta s d h
$$

$\int_{a}^{x} k d h=\beta(x)-\beta(a)$ and $(\beta, k) \in D(h, a, b) ; k$ will be denoted by $\beta^{*}$. Note that $\beta, \alpha, \beta^{*}, p, q$ and $h$ commute on $[a, b]$ and that $q=\beta^{*}+\beta \alpha$.

Proof of $1 \rightarrow 2$. Since the triple $\left(f, f^{*}\right),\left(\beta, \beta^{*}\right), s$ satisfies the hypothesis of Lemma 4.2, then $\left(f \beta, f^{*} \beta+f \beta^{*}+f^{*} s\right) \in$ $D(h, a, b)$. Hence,

$$
\begin{aligned}
\left(f^{*}+f \beta\right. & )^{*}+\left(f^{*}+f \beta\right) \alpha \\
& \cong f^{* *}+f^{*} \beta+f \beta^{*}+f^{*} s+f^{*} \alpha+f \beta \alpha \\
& =f^{* *}+f^{*}(\beta+s+\alpha)+f\left(\beta^{*}+\beta \alpha\right) \\
& =f^{* *}+f^{*} p+f q=r
\end{aligned}
$$

and

$$
\left(f^{*}+f \beta\right)^{*} \cong r-\left(f^{*}+f \beta\right) \alpha
$$

If we integrate each member of the preceding equation with respect to $h$ and recall that $\beta(a)=0$, we obtain 


$$
\left(f^{*}+f \beta\right)(x)=g(x)+(L) \int_{a}^{x}\left(f^{*}+f \beta\right)(-\alpha d h),
$$

where $g(x)=f^{*}(a)+(L) \int_{a}^{x} r d h . \quad$ It follows from Lemma 4.3, $1 \rightarrow 2$, that

$$
\left(f^{*}+f \beta\right)(x)=f^{*}(a)_{a} \prod^{x}(1-\alpha d h)+(R) \int_{a}^{x} d g_{t} \prod^{x}(1-\alpha d h)
$$

for $x \in[a, b]$. Let $w(x)$ respresent the right member in the preceding equation. If $x \in[a, b]$, then $f^{*}(x)=w(x)-f(x) \beta(x)$ and by integrating both members we obtain

$$
f(x)=z(x)+(L) \int_{a}^{x} f(-\beta d h)
$$

where $z(x)=f(a)+(L) \int_{a}^{x} w d h$ and $z(a)=f(a)$. It follows from Lemma $4.3,1 \rightarrow 2$, that

$$
f(x)=f(a){ }_{a} \Pi^{x}(1-\beta d h)+(R) \int_{a}^{x} d z_{t} \Pi^{x}(1-\beta d h) .
$$

Proof of $2 \rightarrow 1$. Functions $f^{* *}$ and $f^{*}$ will be defined such that $\left(f, f^{*}\right)$ and $\left(f^{*}, f^{* *}\right) \in D(h, a, b)$ and such that on $[a, b] f^{* *}+f^{*} p+$ $f q=r$.

Let $f^{*}=w-f \beta$. Since $f$ satisfies the second statement of the conclusion, it follows from Lemma $4.3,2 \rightarrow 1$, that for $x \in[a, b]$

$$
\begin{aligned}
f(x) & =z(x)+(L) \int_{a}^{x} f(-\beta d h) \\
& =f(a)+(L) \int_{a}^{x} w d h+(L) \int_{a}^{x} f(-\beta d h) \\
& =f(a)+(L) \int_{a}^{x} f^{*} d h
\end{aligned}
$$

and $\left(f, f^{*}\right) \in D(h, a, b)$.

Let $f^{* *}$ be the function such that

$$
f^{* *}=r-\left(f^{*}+f \beta\right) \alpha-\left(f^{*} \beta+f \beta^{*}+f^{*} s\right) .
$$


Since $\beta(a)=0$ and

$$
\begin{aligned}
\left(f^{*}+f \beta\right)(x) & =w(x) \\
& =f^{*}(a){ }_{a} \prod^{x}(1-\alpha d h)+(R) \int_{a}^{x} d g_{t} \prod^{x}(1-\alpha d h)
\end{aligned}
$$

for $x \in[a, b]$, it follows from Lemma $4.3,2 \rightarrow 1$, that

$$
\left(f^{*}+f \beta\right)(x)=g(x)+(L) \int_{a}^{x}\left(f^{*}+f \beta\right)(-\alpha d h)
$$

and, hence,

$$
f^{*}(x)=g(x)+(L) \int_{a}^{x}\left(f^{*}+f \beta\right)(-\alpha d h)-f(x) \beta(x) .
$$

Since $\left(f \beta, f^{*} \beta+f \beta^{*}+f^{*} s\right) \in D(h, a, b)$ and $\beta(a)=0$, it follows from the definition of $f^{* *}$ that

$$
\begin{aligned}
(L) \int_{a}^{x} f^{* *} d h & =(L) \int_{a}^{x}\left[r-\left(f^{*}+f \beta\right) \alpha-\left(f^{*} \beta+f \beta^{*}+f^{*} s\right)\right] d h \\
& =-f^{*}(a)+\left[g(x)+(L) \int_{a}^{x}\left(f^{*}+f \beta\right)(-\alpha d h)\right. \\
& =f^{*}(x)-f^{*}(a)
\end{aligned}
$$

for $x \in[a, b]$; hence, $\left(f^{*}, f^{* *}\right) \in D(h, a, b)$.

Since

$$
\begin{aligned}
f^{* *}+f^{*} p+f q= & {\left[r-\left(f^{*}+f \beta\right) \alpha-\left(f^{*} \beta+f \beta^{*}+f^{*} s\right)\right] } \\
& +f^{*}(\alpha+\beta+s)+f\left(\beta^{*}+\alpha \beta\right)=r
\end{aligned}
$$

then the triple $f, f^{*}, f^{* *}$ satisfies the given equation.

Suppose that on $[a, b]$ the functions $h, p$ and $q$ are defined as in Theorem 4.4 except for the restrictions pertaining to $v^{-1}$. If $h \in C^{0}$, it follows from Theorem 3.5 that there is a subdivision $\left\{x_{i}\right\}_{0}^{n}$ of $[a, b]$ and functions $\left\{\beta_{i}\right\}_{1}^{n}, \quad\left\{u_{i}\right\}_{1}^{n}$ and $\left\{v_{i}\right\}_{1}^{n}$ such that for $i=1,2, \cdots, n$ and $x \in\left[x_{i-1}, x_{1}\right]$

$$
\left[u_{i}(x), v_{i}(x)\right]=[0,1]_{x_{t-1}} \Pi^{x}\left(I+\left[\begin{array}{rr}
-p & -1 \\
q & 0
\end{array}\right] d h\right),
$$


$\beta_{i}(x)=v_{i}(x)^{-1} u_{i}(x)$, and $v_{i}^{-1}$ exists and is bounded on $\left[x_{i-1}, x_{i}\right]$. Hence, for $i=1,2, \cdots, n$, Theorem 4.4 gives the solution of $f^{* *}+f^{*} p+f q=r$ on $\left[x_{i-1}, x_{i}\right]$ which is unique for a given pair $f^{*}\left(x_{i-1}\right)$ and $f\left(x_{i-1}\right)$. Therefore, Theorem 4.4 can be used to find a unique solution on $[a, b]$ for given values of $f(a)$ and $f^{*}(a)$.

A theorem similar to Theorem 4.4 can be stated and proved for the equation $f^{* *}+p f^{*}+q f=r$; however, Theorem 5.2 of [3] would be used in the proof instead of Lemma 4.3. If $\left(f, f^{*}\right)$ means $f(x)-f(a)=$ (R) $\int_{a}^{x} f^{*} d h$ and $h$ is right continuous, a theorem similar to Theorem 4.4 can be stated and proved.

\section{REFERENCES}

1. P. Puig Adam, Los fracciones continuas de cocientes incompletos differenciales y sus aplicaciones, Revista Mat. Hisp. Amer. II, (1951), 180-190.

2. W. D. L. Appling, Interval functions and real Hilbert spaces, Rend.-Circl. Mat. Palermo, Ser. 2, 11 (1962), 154-156.

3. B. W. Helton, Integral equations and product integrals, Pacific J. Math. 16 (1966), 297-322.

4. - A product integral representation for a Gronwall inequality, Proc. Amer. Math. Soc., 23 (1969), 493-500.

5. - Solutions of $f(x)=f(a)+(R L) \int_{a}^{x}(f H+f G)$ for rings, Proc. Amer. Math. Soc., 25 (1970), 735-742.

6. Jon C. Helton, An existence theorem for sum and product integrals, Proc. Amer. Math. Soc., 39 (1973), 149-154.

7. J. S. MacNerney, Stieltjes integrals in linear spaces, Ann. of Math., (2) 61 (1955), 354-367.

8. - Continuous products in linear spaces, J. Elisha Mitchell Sci. Soc., 71 (1955), 185-200.

9. - Integral equations and semigroups, Illinois J. Math., 7 (1963), 148-173.

10. Alfred Stohr, Über einen integralartigen Grenzubergang bei Kettenbruchen, Math. Nachr., 6 (1951), 103-107.

11. H. S. Wall, Concerning continuous continued fractions and certain systems of Stieltjes integral equations, Rend. Circ. Mat. Palermo, (2), 2 (1953), 73-84.

12. - Concerning harmonic matrices, Archiv. der Math., V (1954), 160-167.

Received September 12, 1973 and in revised form June 11, 1974.

Southwest Texas State University 



\section{Pacific Journal of Mathematics}

\section{Vol. 56, No. $1 \quad$ November, 1975}

Shimshon A. Amitsur, Central embeddings in semi-simple rings .......... 1

David Marion Arnold and Charles Estep Murley, Abelian groups, A, such

that $\operatorname{Hom}(A,---)$ preserves direct sums of copies of $A \ldots \ldots \ldots .$.

Martin Bartelt, An integral representation for strictly continuous linear

operators ................................... 21

Richard G. Burton, Fractional elements in multiplicative lattices......... 35

James Alan Cochran, Growth estimates for the singular values of

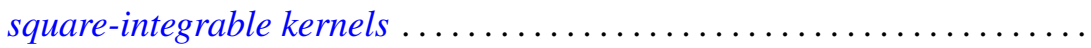

C. Martin Edwards and Peter John Stacey, On group algebras of central

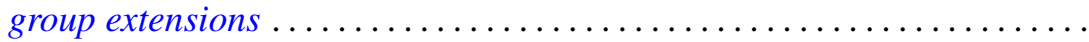

Peter Fletcher and Pei Liu, Topologies compatible with homeomorphism

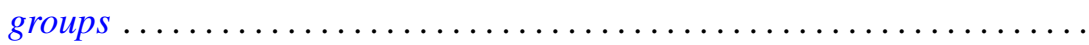

George Gasper, Jr., Products of terminating ${ }_{3} F_{2}(1)$ series ............ 87

Leon Gerber, The orthocentric simplex as an extreme simplex ............

Burrell Washington Helton, A product integral solution of a Riccati

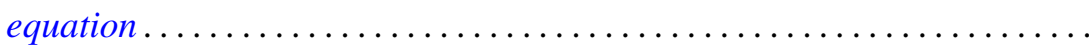

Melvyn W. Jeter, On the extremal elements of the convex cone of

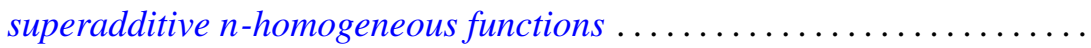

R. H. Johnson, Simple separable graphs .

Margaret Humm Kleinfeld, More on a generalization of commutative and

alternative rings. . .

A. Y. W. Lau, The boundary of a semilattice on an $n$-cell.

Robert F. Lax, The local rigidity of the moduli scheme for curves ...

Glenn Richard Luecke, A note on quasidiagonal and quasitriangular

operators .

Paul Milnes, On the extension of continuous and almost periodic functions

Hidegoro Nakano and Kazumi Nakano, Connector theory.

James Michael Osterburg, Completely outer Galois theory of perfect rings ..................................

Lavon Barry Page, Compact Hankel operators and the F. and M. Riesz

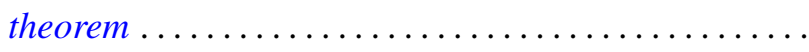

Joseph E. Quinn, Intermediate Riesz spaces..................... 225

Shlomo Vinner, Model-completeness in a first order language with a generalized quantifier.

Jorge Viola-Prioli, On absolutely torsion-free rings ..........

Philip William Walker, A note on differential equations with all solutions of integrable-square............................

Stephen Jeffrey Willson, Equivariant maps between representation 